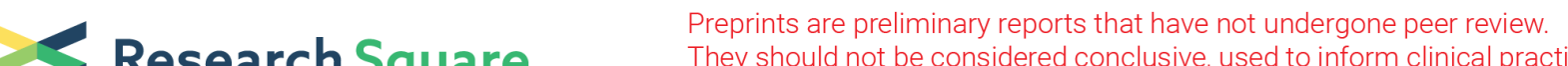 Research Square They should not be considered conclusive, used to inform clinical practice, or referenced by the media as validated information.
}

\section{Isolated Congenital Cleft Mitral Valve Leaflet: A Rare Cause of Refractory Cardiogenic Shock Complicating Acute Myocardial Infarction}

Mubashir Hamid Bahrami ( $\square$ mhbahram@iu.edu)

Indiana University School of Medicine https://orcid.org/0000-0002-5734-6876

Hamza Ansari

Indiana University School of Medicine

Maya Guglin

Indiana University School of Medicine, Indiana University Health Cardiology Physicians

Georges Ephrem

Indiana University School of Medicine, Indiana University Health Cardiology Physicians

George Revtyak

Indiana University School of Medicine, Indiana University Health Physicians Cardiology

\section{Case Report}

Keywords: Cleft mitral valve, Congenital heart disease, Mitral regurgitation, MitraClip, Cardiogenic shock, Acute myocardial infarction, Mechanical circulatory support, Transesophageal echocardiography

Posted Date: December 9th, 2020

DOI: https://doi.org/10.21203/rs.3.rs-121704/v1

License: (1) This work is licensed under a Creative Commons Attribution 4.0 International License.

Read Full License 


\section{Abstract}

We report a unique presentation of isolated congenital cleft mitral valve complicating cardiogenic shock from acute myocardial infarction. Isolated cleft mitral valve is an uncommon diagnosis that can have significant clinical implications, especially if not recognized in patients presenting to the catheterization lab with acute myocardial infarction and cardiogenic shock. A review of this rare diagnosis including the options and timing of therapeutic interventions, which can include MitraClip, is important for publication.

The case is of a patient who presented with an anterior acute ST elevation myocardial infarction. Despite early coronary revascularization and conventional support, refractory cardiogenic shock ensued requiring mechanical circulatory support escalation to Veno-arterial extracorporeal membrane oxygenation. Subsequently, left ventriculography revealed a massively dilated left atrium and severe mitral regurgitation raising concerns for a mechanical mitral valve complication. The patient was taken to the operating room for possible mitral valve surgery, but a preoperative transesophageal echocardiogram revealed an isolated posterior cleft mitral valve. Since the patient had stabilized on mechanical circulatory support, emergent surgery was deferred. The patient successfully recovered during index hospitalization with mechanical circulatory support and discharged on guideline directed medical therapy.

In conclusion, isolated cleft mitral valve is a rare diagnosis that can often be underrecognized without comprehensive 3-dimensional transesophageal echocardiography evaluation. If diagnosed early with significant regurgitation, surgical treatment results in good outcomes and preservation of LV systolic function. Percutaneous correction of a CMVL with MitraClip has been described and may offer an alternative approach for high risk surgical patients.

\section{Background}

Cleft mitral valve leaflet (CMVL) is the most common cause of congenital mitral regurgitation (MR). CMVL results from congenital mitral hypoplasia and is most often associated with other congenital heart defects, usually endocardial cushion defects (atrio-ventricular septal defects) ${ }^{(1,2)}$. Cases in which CMVL is found without an associated defect are called isolated mitral valve clefts, which is another rare congenital anomaly that was first described in $1954{ }^{(3)}$. Both isolated anterior and posterior mitral leaflet clefts have been reported in the literature. In younger patients, CMVL commonly causes only mild MR and is often not recognized, but severity often increases with age MR ${ }^{(3)}$.

This case describes a patient presenting with acute myocardial infarction (AMI) who, despite early emergent revascularization, developed refractory cardiogenic shock (CS) necessitating mechanical circulatory support (MCS). The patient was ultimately found to have an isolated posterior CMVL and severe MR, which was managed without emergent surgical intervention and resulted in early patient recovery. The patient's long-term course for his CMVL and MR was more complex, and it remained a question of whether or not to repair his defect and the ideal timing of intervention should it be pursued. 


\section{Case Presentation}

A 49-year-old Caucasian male truck driver with no prior medical history was driving through the state when he developed sudden onset chest pain. Upon arrival to the ER, his electrocardiogram showed rapid atrial fibrillation with ST elevations in the anterior precordial leads. He was hypotensive with initial blood pressure of $90 / 70 \mathrm{mmHg}$ and hypoxic requiring supplemental oxygen.

Emergent coronary angiography revealed acute occlusion of the ostial left anterior descending artery (LAD) with TIMI grade 0 flow (Supplementary Video 1). Using a workhorse wire, the lesion was crossed and aspiration thrombectomy performed using the Export AP aspiration catheter, restoring TIMI 2 blood flow. The patient became progressively more hypotensive and hypoxic requiring intubation and initiation of vasopressors. Following stabilization on vasopressors, percutaneous coronary intervention (PCl) was successfully performed using aspiration thrombectomy and drug eluting stent placement with restoration of TIMI 3 flow (Supplementary Video 2). Despite revascularization, worsening shock ensued requiring intra-aortic balloon pump insertion and increasing doses of dopamine $(20 \mathrm{mcg} / \mathrm{kg} / \mathrm{min})$ and norepinephrine $(20 \mathrm{mcg} / \mathrm{min})$.

Left heart catheterization showed a persistently narrow pulse pressure $(20 \mathrm{mmHg})$ and an elevated left ventricular end-diastolic pressure (LVEDP) of $33 \mathrm{mmHg}$ (Figure 1). Left ventriculography and aortography revealed severe (4+) MR with pulmonary vein opacification and a massively enlarged left atrium (Figure 2). No aortic regurgitation or aortic dissection was detected.

With continually worsening CS, MCS was escalated to Veno-arterial extracorporeal membrane oxygenation (VA-ECMO) in the cardiac catheterization lab. On VA-ECMO, the patient stabilized and was taken to the operating room for possible mitral valve surgery because of concerns for an acute mechanical mitral valve complication. A pre-operative transesophageal echocardiography (TEE) revealed markedly reduced global LV systolic function, an isolated cleft posterior mitral valve leaflet (CMVL) and severe MR (Figure 3, Supplementary Video 3, Supplementary Video 4). As the patient was favorably responding to VA-ECMO, surgery was not performed, and the patient was managed medically. His clinical improvement was rapid, and vasopressors were completely weaned by hospital day 5. VA-ECMO was decannulated on day 7. Somewhat surprisingly, repeat transthoracic echocardiography revealed only mild MR with a LV ejection fraction of $45 \%$.

Following extubation, the patient mentioned having a "heart murmur" that had never been fully evaluated or treated. He was discharged fully ambulatory on hospital day 10 with guideline-directed medical therapy. Unfortunately, he had multiple "heart failure" hospitalizations over the next 6 months and ultimately presented with low output heart failure, severe MR and severe global LV systolic dysfunction (LAD still patent by coronary angiography). He eventually received a HeartMate 3 Left Ventricular Assist Device (LVAD) which dramatically reduced the MR and resulted in clinical stability.

\section{Discussion}


Although an isolated CMVL is an uncommon congenital cardiac diagnosis, it is a well-recognized cause of congenital MR. Isolated CMVL should be distinguished from cleft mitral valves that are associated with atrioventricular septal defects or other forms of congenital heart disease. It is rare to find an isolated CMVL in either the anterior or posterior leaflet, with one study using transthoracic echocardiography reporting a prevalence of isolated posterior CMVL with moderate or severe MR of $0.07 \%{ }^{(4)}$. A retrospective, unpublished review of the echocardiogram reports from 2011 through 2020 at our institution showed a total of 24 patients with documented cleft mitral valve. Of these cases, $91.7 \%$ (22 out of 24 patients) had some form of associated atrioventricular septal defects, often completely repaired earlier in life. Anterior clefts were much more common than posterior clefts, 21 and 3 clefts, respectively. Another study reviewed 19 consecutive patients with cleft mitral valve on echocardiography and found that 15 of the patients had an associated congenital heart lesion (atrial septal defect, ventricular septal defect, patent ductus arteriosus or tetralogy of Fallot) ${ }^{(1)}$. Of the 4 patients with isolated CMVL in this study, only 1 patient had a posterior CMVL. CMVL can sometimes be associated with additional mitral valve abnormalities including generalized thickening of leaflets, prolapse of anterior leaflet, papillary muscle anomalies, and anomalous insertion of the anterior leaflet chordae (usually towards the LV outflow tract sometimes causing the obstruction) ${ }^{(5)}$. Functional consequences of CMVL can include significant MR and less commonly LV outflow tract obstruction in the presence of abnormal chordal attachments ${ }^{(6)}$.

The diagnosis of CMVL is commonly made my echocardiography, particularly TEE with 3-D acquisition (7). While an isolated CMVL is rare, it must be acknowledged that the diagnosis of CMVL using transthoracic echocardiography is particularly challenging and often leads to under recognition. As such, the increasing use of 3-D TEE has resulted in better understanding of the mechanisms of hemodynamically significant MR (including CMVL) before surgical or percutaneous intervention (7-9). When patients with significant MR due to unrecognized CMVL are referred for surgical intervention, the likelihood of its diagnosis in a non-beating, flaccid heart at the time of surgery is low. A pre-operative TEE, as was performed in our patient's case, can be very helpful with establishing a diagnosis as well as planning and assessing the feasibility of repair.

Surgical repair of a mitral cleft is usually the treatment of choice. Surgical repair is most often completed by suturing opposed edges of the cleft coupled with annuloplasty. When there is a lack of substance due to fibrous shrinkage of the valvular tissue, resection of the cleft edges together with partial tissue replacement using an autologous pericardial tissue can accomplish a stable valve reconstruction $(5,10)$.

Percutaneous intervention with Mitraclip has also been used to successfully treat CMVL when the valvular anatomy appears favorable ${ }^{(11)}$. Willemsen et. al. described the first use of MitraClip in a patient with severe MR and dilated cardiomyopathy who also had an isolated posterior CMVL ${ }^{(12)}$. In this case, a MitraClip was placed posteromedial right along the cleft, which reduced the MR in the cleft. However, there remained a new MR jet in the more anterolateral side, so a second MitraClip was placed on the anterolateral side in symmetric fashion with a significant reduction in MR. Furthermore, MitraClip may be 
a safer option for high surgical risk patients or those with previously repaired atrioventricular septal defects who experience progressive MR later in life ${ }^{(11,13)}$.

Another interesting feature of this case is the dynamic nature of severe MR, which was likely a major contributor to the refractory CS. Completely undiagnosed and not causing any symptoms prior to the AMI, the MR became severe in the setting of anterior wall stunning and increased afterload from high-dose vasopressors. This prompted an urgent evaluation of the mitral valve for potential surgical repair. The severity of MR dramatically decreased after several days of support, but then increased again as left ventricular remodeling progressed over following months. Only the durable LVAD eventually stabilized the patient and resulted in a marked reduction of the MR which typically occurs with mechanical left ventricular unloading, especially in HeartMate $3^{(14)}$.

In retrospect, the patient in our case may have missed a window of opportunity for repair of his CMVL (with associated MR), prior to developing progressive LV dilation and end-stage heart failure. The severe, refractory CS experienced by our patient was disproportionate to the degree of LV systolic dysfunction immediately post LAD revascularization. This suggests poor cardiac reserve as a result of longstanding MR caused by the underlying, isolated posterior CMVL. Given the ultimate hemodynamic consequences of his CMVL and MR, one can argue that this patient's long-term course may have been more favorably altered if this were corrected prior to discharge. Because of the massively dilated LA, evaluation with only transthoracic echocardiography might significantly underestimate the true functional severity of MR. In this case, full hemodynamic assessment in the cardiac catheterization laboratory prior to hospital discharge would provide better clinical data upon which to base therapeutic decision making, especially regarding invasive MV corrective therapies.

\section{Conclusions}

Isolated CMVL is a rare disorder, especially in the posterior leaflet. The consequences of CMVL can include significant mitral regurgitation, left atrial enlargement, and progressive LV dilation. If diagnosed early, surgical treatment results in good outcomes and preservation of LV systolic function. Percutaneous correction of a CMVL with MitraClip has been described and may offer an alternative approach for high risk surgical patients. Furthermore, early identification of CS in AMI is critically important to determine escalation of treatment and implementation of mechanical circulatory support to reduce overall morbidity/mortality. Additional hemodynamic and angiographic data (LVEDP, left ventriculography, aortography, and TEE with three-dimensional acquisition) remain essential to fully evaluate patients with CS complicating AMI, especially if a mechanical complication is suspected.

\section{Abbreviations}

$\mathrm{AMI}=$ Acute myocardial infarction

CMVL $=$ Cleft mitral valve leaflet 
CS = Cardiogenic shock

IABP = Intraaortic balloon pump

$L A D=$ Left anterior descending

LVEDP = Left ventricular end-diastolic pressure

MCS = Mechanical circulatory support

$\mathrm{MR}=$ Mitral Regurgitation

$\mathrm{PCl}=$ Percutaneous coronary intervention

LVAD = Left Ventricular Assist Device

TEE $=$ transesophageal echocardiography

$\mathrm{TIMI}=$ Thrombolysis in myocardial infarction

VA-ECMO $=$ Veno-arterial extracorporeal membrane oxygenation

\section{Declarations}

- Ethics approval consent to participate: Not applicable

- Consent for publication: The individual patient is willing to consent

- Availability of data and materials: Data sharing is not applicable to this case report as no datasets were generated or analyzed

- Competing interests: The authors declare that they have no competing interests

- Funding: No declarable source of funding for this manuscript

- Author's contributions: All authors contributed to the written text and editing in final preparation of this manuscript

- Acknowledgements: none

\section{References}

1. Yuan X, Zhou A, Chen L, Zhang C, Zhang Y, Xu P. Diagnosis of mitral valve cleft using real-time 3dimensional echocardiography. J Thorac Dis. 2017;9(1):159-165.

2. Tamura M, Menahem S, Brizard C. Clinical Features and Management of Isolated Cleft Mitral Valve in Childhood. J Am Coll Cardiol 2000;35:764 -770.

3. Timóteo A, Galrinho A, Fiarresga A, Branco L, Banazol N, Leal A, Fragata J, Quininha J. Isolated cleft of the anterior mitral valve leaflet. European Journal of Echocardiography 2007; 8(1):59-62. 
4. Kuijpers JM, Vaartjes I, Bokma JP, et al. Risk of coronary artery disease in adults with congenital heart disease: A comparison with the general population. Int J Cardiol. 2020 Apr 1;304:39-42.

5. Perier P, Clausnizer B. Isolated Cleft Mitral Valve: Valve Reconstruction Techniques. Ann Thorac Surg 1995: 59:56-59.

6. Du X, Huang C, Wan Z. Successful mitral valve repair for severe mitral regurgitation caused by isolated cleft of the posterior leaflet. Int J Cardiol 2009; 150(2):e48-9

7. Narang A, Addetia K, Weinert L, Yamat M, Shah AP, Blair JE, Mor-Avi V, Lang RM. Diagnosis of Isolated Cleft Mitral Valve Using Three-Dimensional Echocardiography. J Am Soc Echocardiogr. 2018 Nov;31(11):1161-1167.

8. Wyss CA, Enseleit F, van der Loo B, Gr€unenfelder J, Oechslin EN, Jenni R. Isolated cleft in the posterior mitral valve leaflet: a congenital form of mitral regurgitation. Clin Cardiol 2009;32:553-60.

9. Moura-Ferreira S, Sampaio F, Ribeiro J, Fontes-Carvalho R. A rare case series of mitral valve clefts diagnosed by 3D echocardiography and mini-review of the literature. Echocardiography. 2019 Jun;36(6):1203-1207.

10. Zhu, Diqi et al. "Isolated cleft of the mitral valve: clinical spectrum and course." Texas Heart Institute journal 36,6 (2009): 553-6.

11. Melillo E, Ancona F, Buzzatti N, Denti P, Agricola E. A challenging mitral valve anatomy for percutaneous repair with MitraClip: cleft posterior leaflet, Eurt Heart J - Cardiovascular Imaging 2019; 20 (12): 1433-1434.

12. Hendrik Martinus Willemsen, Ad van den Heuvel, Remco Schurer, Joost van Melle, Eshan Natour, Mitral cleft repair by mitraclipping, European Heart Journal, Volume 35, Issue 16, 21 April 2014, Page 1021.

13. Cheng R, Kar S, Siegel RJ, Nakamura M. Cleft mitral leaflets and severe MR: Testing the limits of percutaneous mitral valve repair. Catheter Cardiovasc Interv. 2019 May 1;93(6):1161-1164.

14. Kanwar MK, Rajagopal K, Itoh A, Silvestry SC, Uriel N, Cleveland Jr JC, Salerno CT, Horstmanshof D, Goldstein DJ, Naka Y, Bailey S. Impact of left ventricular assist device implantation on mitral regurgitation: An analysis from the MOMENTUM 3 trial. The Journal of Heart and Lung Transplantation. 2020 Jun 1;39(6):529-37.

\section{Figures}



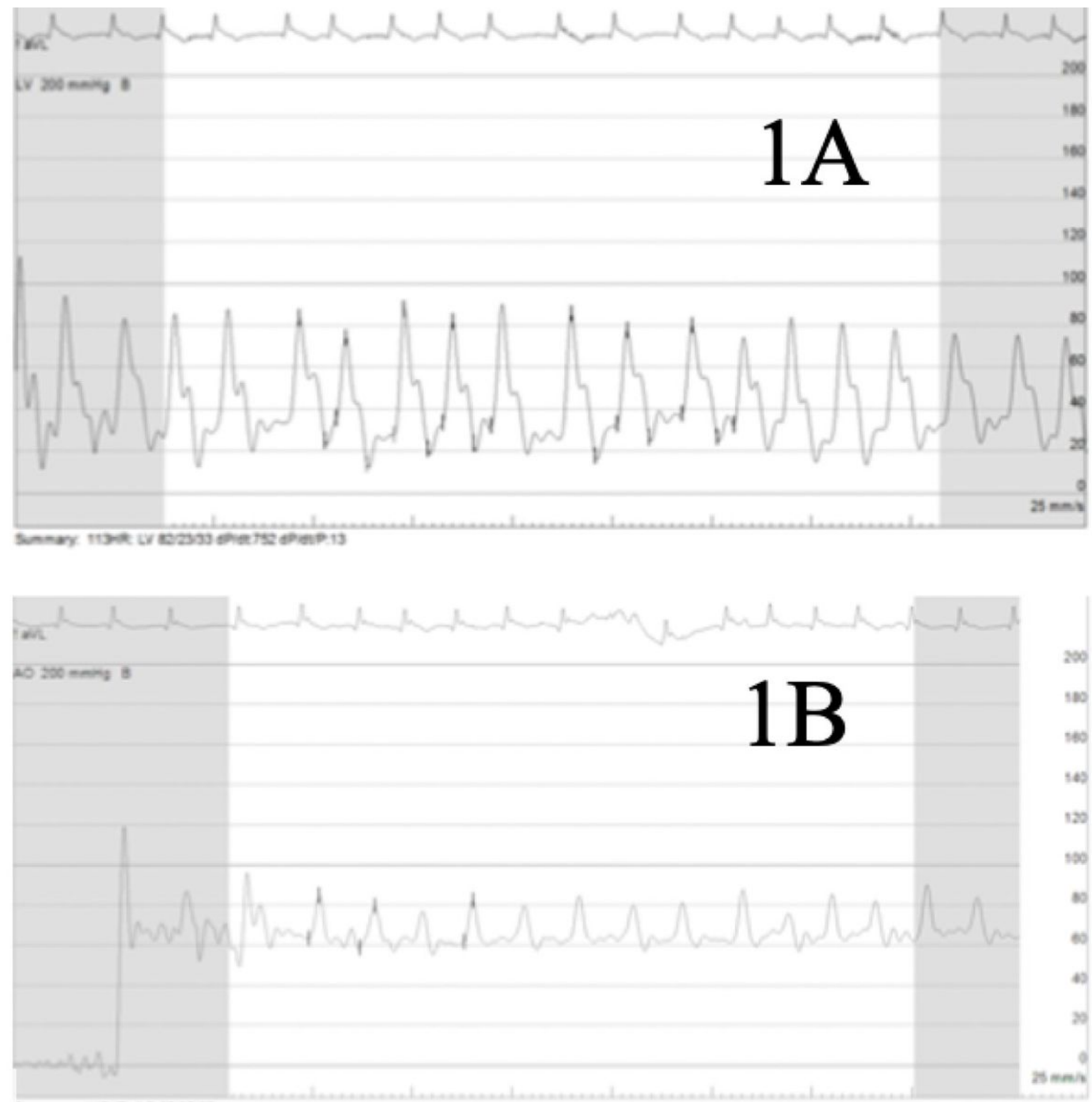

Figure 1

Hemodynamics: A left ventricular hemodynamic tracing post PCI showed elevated LVEDP (Top) and narrow systemic pulse pressure (Bottom). 

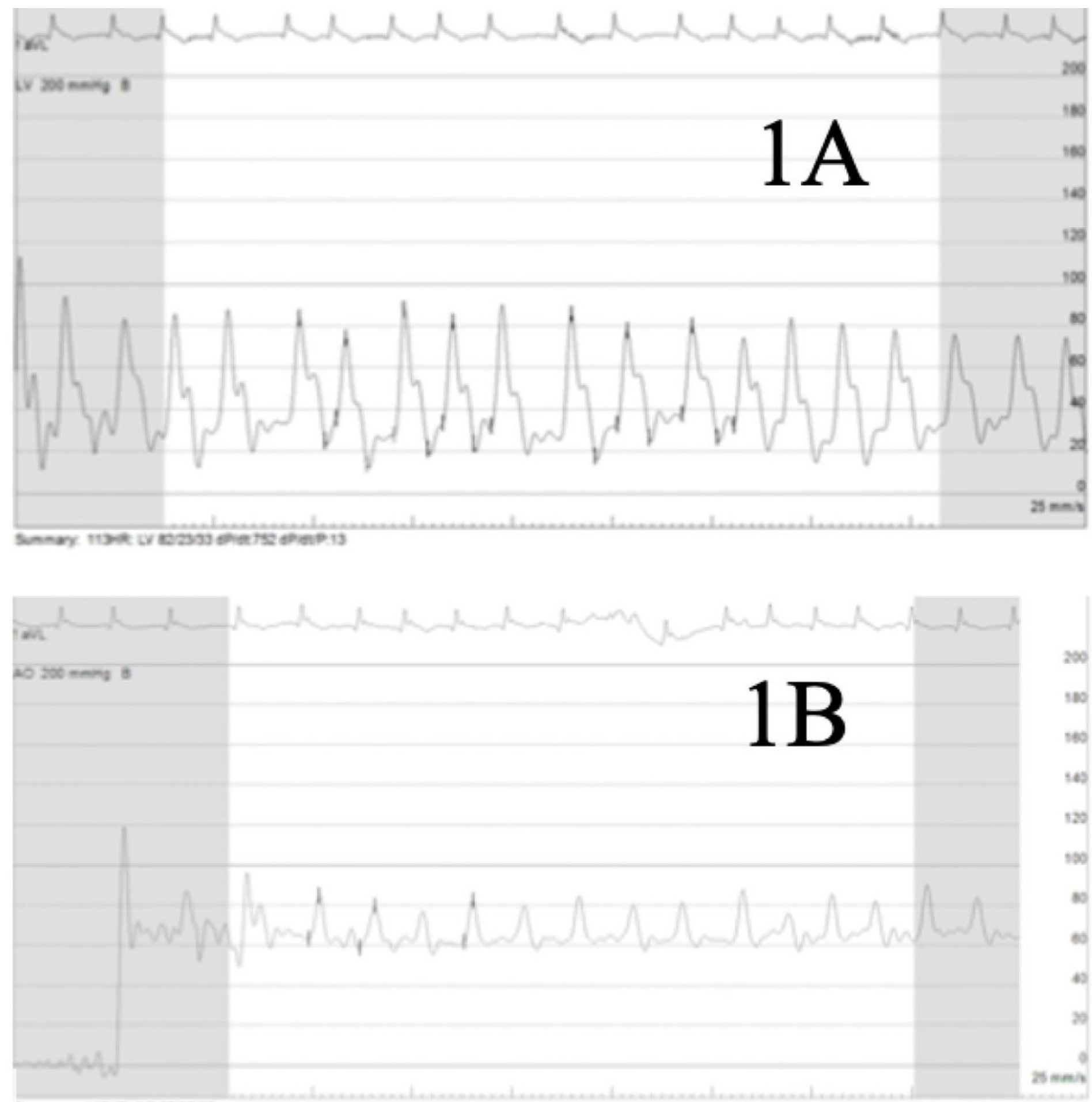

Figure 1

Hemodynamics: A left ventricular hemodynamic tracing post PCI showed elevated LVEDP (Top) and narrow systemic pulse pressure (Bottom). 


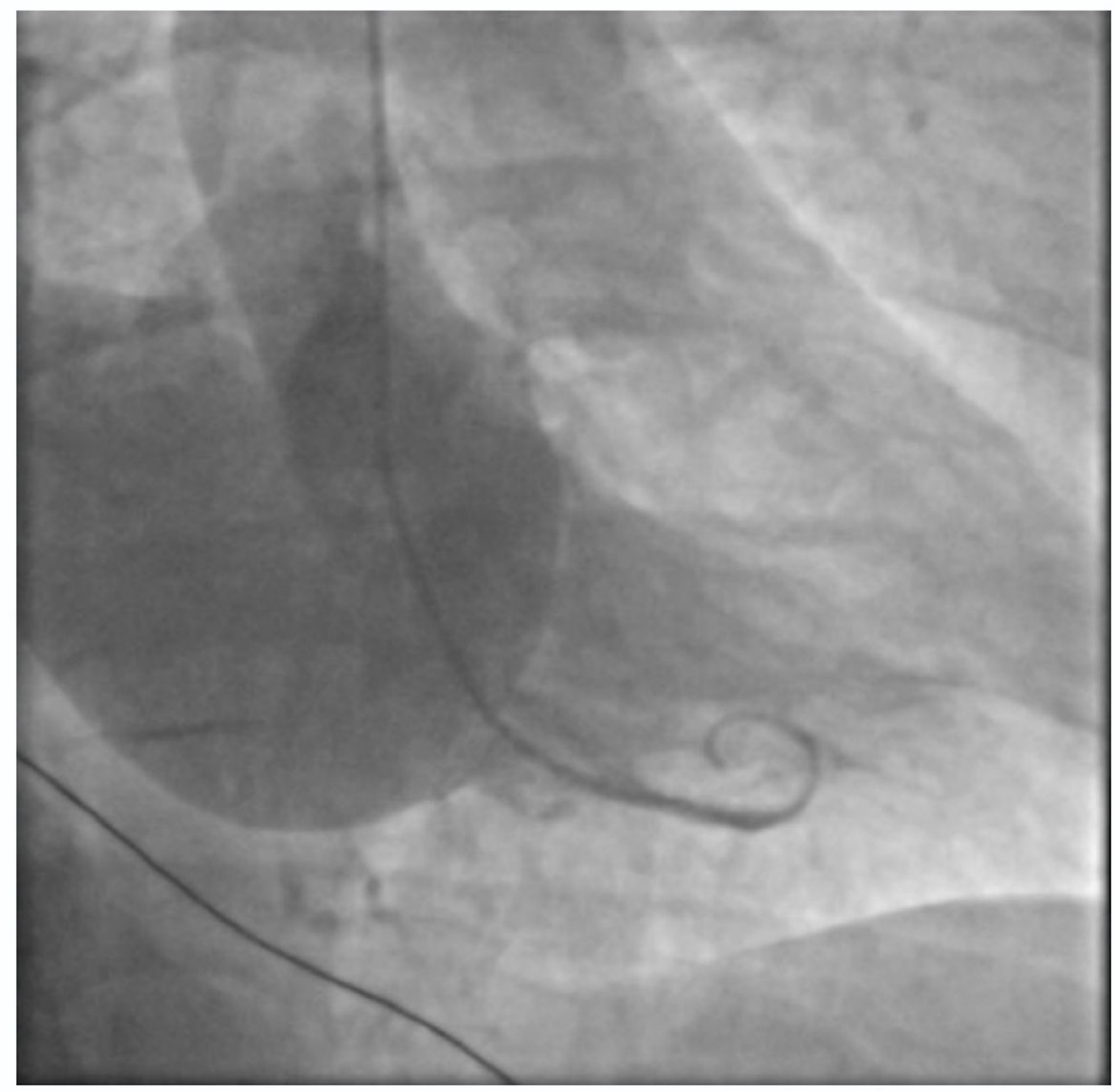

Figure 2

Left Ventriculography: Left ventriculography showing severe (4+) angiographic mitral regurgitation. 


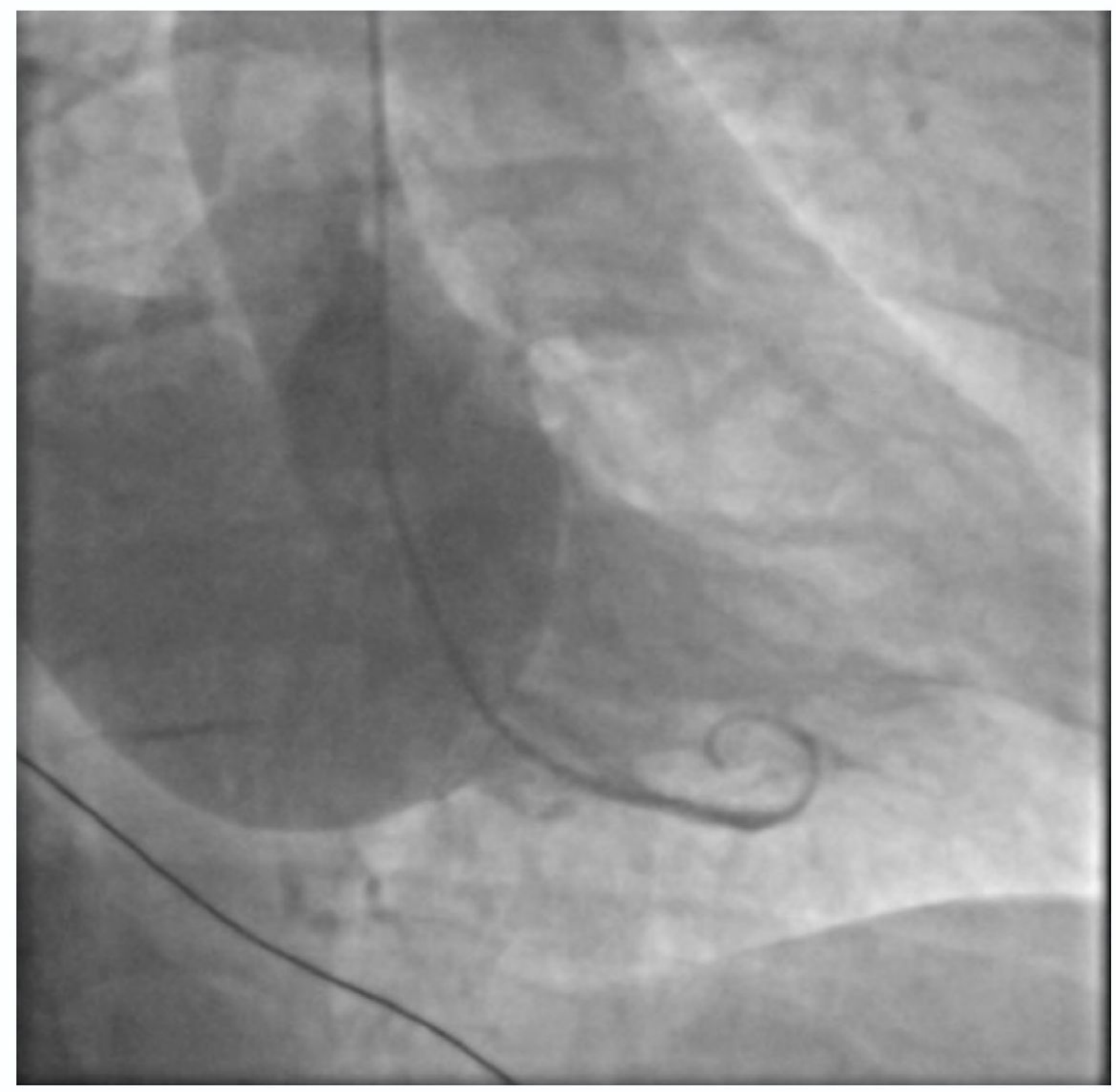

Figure 2

Left Ventriculography: Left ventriculography showing severe (4+) angiographic mitral regurgitation. 


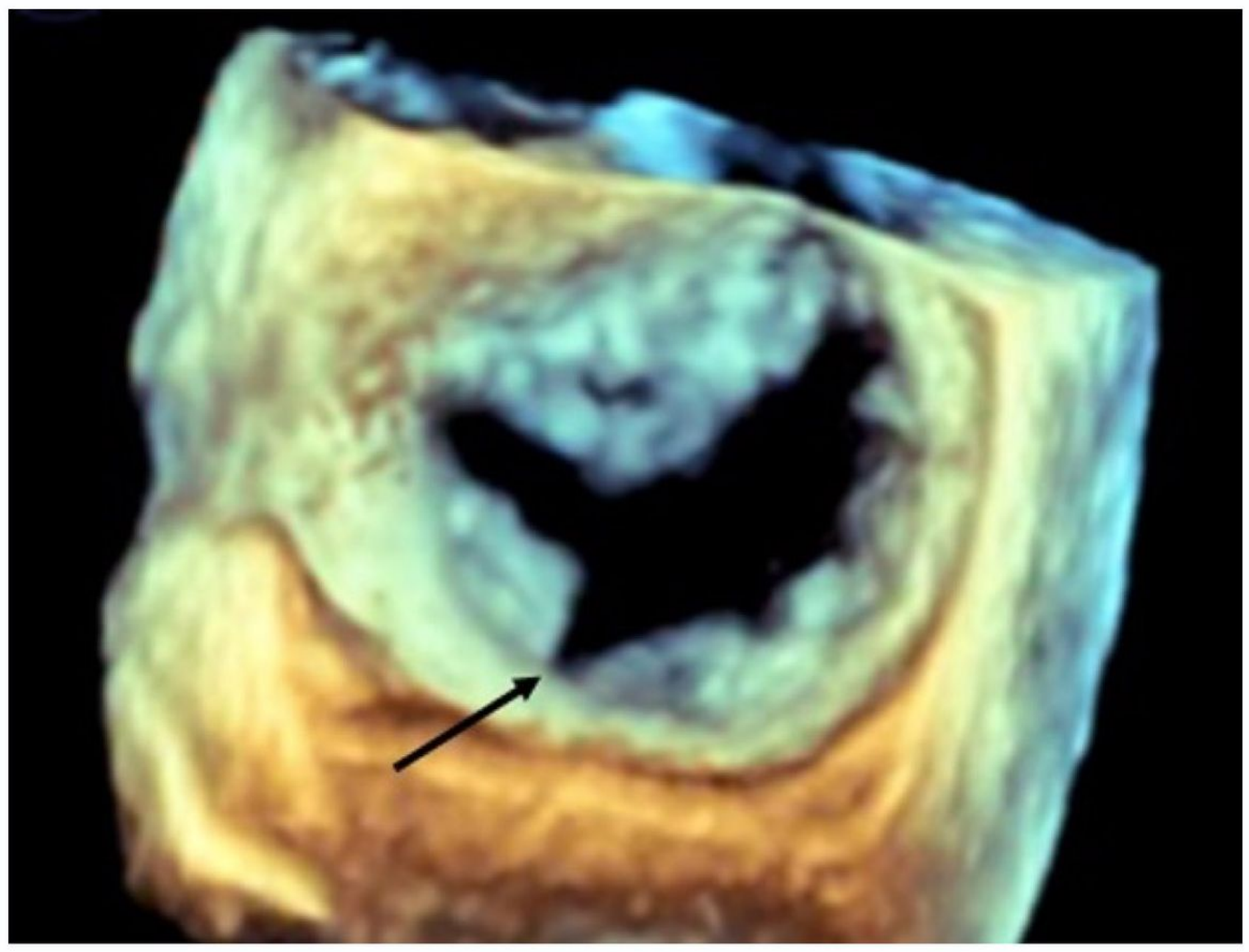

Figure 3

Intraoperative TEE: A three-dimensional TEE image revealed CMVL. 


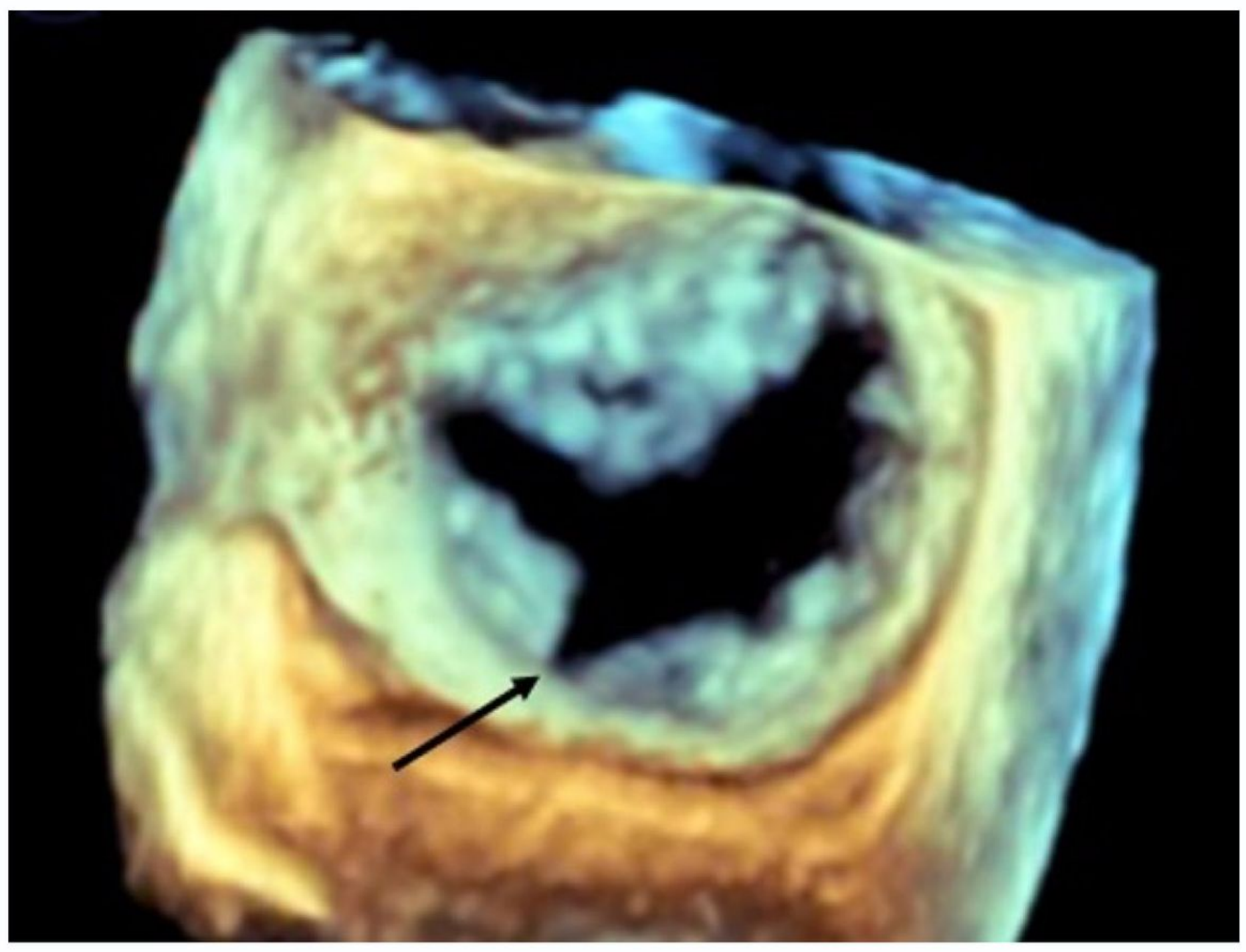

Figure 3

Intraoperative TEE: A three-dimensional TEE image revealed CMVL.

\section{Supplementary Files}

This is a list of supplementary files associated with this preprint. Click to download.

- CoronaryAngiographyPostintervention.mov

- CoronaryAngiographyPostintervention.mov

- Coronaryangiography.mov

- Coronaryangiography.mov

- IntraoperativeTEE2DMitralregurgitation.mov

- IntraoperativeTEE2DMitralregurgitation.mov

- IntraoperativeTEE.mov 
- IntraoperativeTEE.mov

Page 14/14 